\title{
Erratum to: Reinforcement learning and neural networks \\ for multi-agent nonzero-sum games of nonlinear constrained-input systems
}

\author{
Sholeh Yasini • Mohammad Bagher Naghibi Sistani • \\ Ali Karimpour
}

Published online: 18 February 2015

(c) Springer-Verlag Berlin Heidelberg 2015

Erratum to: Int. J. Mach. Learn. \& Cyber.

DOI 10.1007/s13042-014-0300-y

In the original publication of the article, the second and the third author names were incorrectly spelled in the author group. The name should appear as Mohammad Bagher Naghibi Sistani and Ali Karimpour.

The online version of the original article can be found under doi:10.1007/s13042-014-0300-y.

S. Yasini · M. B. Naghibi Sistani $(\bowtie) \cdot$ A. Karimpour Department of Electrical Engineering, Ferdowsi University of Mashhad, Mashhad 91775-1111, Iran

e-mail: mb-naghibi@um.ac.ir

S. Yasini

e-mail: sholeh.yasini@gmail.com; sh.yasini@stu-mail.um.ac.ir 